\title{
SPECIAL SECTION
}

\section{LEISHMANIASIS EPIDEMIOLOGY}

Proceedings of the International Study Workshop on Leishmaniasis Epidemiology held at the International Centre of Insect Physiology and Ecology (ICIPE), Duduville International Guest Centre (DIGC), Nairobi, Kenya

10-13 September 1984 


\title{
PREFACE
}

\section{LEISHMANIASIS}

\section{GLOBAL IMPACT ON HEALTH}

\author{
P. DE RAADT \\ World Health Organization, 1211 Geneva, 27, Switzerland
}

(Received 13 August 1985)

\section{PREVALENCE}

Over the last decade, public authorities from all parts of the world have expressed their growing concern about leishmaniasis being a serious threat to health. This increased awareness occurred partly as a result of serious outbreaks which were reported, such as for instance, the one in Bihar, India and earlier in Kenya. Several health services in South America have indicated increases in both the mucocutaneous and cutaneous disease. It is difficult to say to what extent these observations reflect true increases or perhaps indicate more successful efforts in detecting new patients.

On the African continent, south of the Sahara, in Ethiopia, Kenya and Sudan, leishmaniasis foci have for a long time been identified. During the last 15 years, an increasing number of sporadic new infections in man were reported from practically all African countries. The significance of these sporadic infections is not certain: they may be locally acquired with a curiously low prevalence, or they may reflect unawareness of the local health services and lack of diagnostic experience, or they may have been imported cases. Certainly several potential transmission sites occur outside the three well-known endemic countries and, where possible, such areas should receive the attention of research or control groups to determine if any Leishmania are circulating.

\section{EPIDEMIOLOGY}

In spite of the alarming indications of accelerated transmission and the spread of leishmaniasis in the world, the complex nature of studying the local epidemiology prevents many health services from developing an appropriate control programme. The difficulties start already at data collection and reporting. Reliable diagnosis of visceral leishmaniasis, for instance, is often beyond the capacity of the rural health centres to where the majority of new patients refer themselves. The clinical signs are not sufficiently specific and cannot easily be differentiated from febrile conditions such as malaria, typhoid fever, brucellosis and many others. Splenic punctures or serology require technical skill and equipment which is rarely available in the endemic areas. To this is added that leishmaniasis, at least in Africa, is not obligatorily notifiable which is a second reason why the prevalence data are unreliable.

\section{TRANSMISSION}

The wide range of varieties of transmission cycles is another obstacle: the interrelated transmission cycles involving man, wild animals and domestic animals are in themselves not exceptional and are similar to diseases such as African trypanosomiasis and American trypanosomiasis. In leishmaniases, however, the variety of vectors and reservoir hosts requires detailed epidemiological analysis each time, before control can be planned.

\section{The facts speak for themselves}

According to presently available data amongst the 112 countries listed in the world, known or suspected to have one or more foci of transmission, in only 18 were studies carried out to identify reservoir hosts and in 11 countries the vector was incriminated on the basis of solid evidence.

The paradox is easily explained when realizing that in many of the leishmaniasis reporting countries, the average expenditure on health is less than U.S.\$10 per person per year. Under such tight budgetary constraints, there is hardly room for field research on vectors and animal reservoirs nor for bloodmeal analysis or parasite identification.

Since, in many of the countries where leishmaniasis is endemic, it is unlikely that the resources in manpower and finances required for such field research will sufficiently increase in the immediate future, there is a case for technically accepting certain compromises, at present applied, from force of circumstances, by health services in an attempt to optimize available resources.

\section{CONTROL}

The minimum standard of control is (1) passive surveillance only for detection and treatment without preventive measures. Here, joint international and national efforts can be made to improve case detection by involving primary health care workers in 
identifying suspects, by improving facilities in the treatment centres for confirmation of diagnosis or, in the case of visceral leishmaniasis, establishing a centre for serodiagnosis, and finally, ensuring appropriate drug supplies to the treatment centres. In slightly better conditions, (2) active surveillance of the human populations can be added to define geographic boundaries and obtain an idea of the rate of transmission. The next level of improvement, (3) would then be to arrange field studies and sample potential reservoir animals and vectors. Finally, (4) design a control programme based on providing curative treatment and adequate preventive measures.

Most of the current techniques applicable during these sequential steps have been recently reviewed (WHO, 1984) at a global level. In order to facilitate establishment or reinforcement of leishmaniasis control programmes, there is a need for regional reviews to identify common disease manifestations and types of transmission which are characteristic for certain subregions or continents. By such analysis, options for control strategies can be narrowed down to a limited number of stereotype control approaches applicable in given geographic areas.

The contents of this issue of Insect Science and Its Application presents the scientific papers and recommendations made at the International Study Workshop on Leishmaniasis Epidemiology, held in Nairobi, 10-13 September 1984 and organized by the International Centre of Insect Physiology and Ecology (ICIPE), Nairobi. It is hoped that this review, with particular reference to the situation in Africa, will contribute to strengthening international collaboration between the leishmaniasis research workers in Africa and mobilizing expertise betweep the countries with leishmaniasis problems in order to make maximum use of the resources available.

\section{REFERENCE}

WHO (1984) The Leishmaniases. WHO Technical Report Series No. 70I, 1984. WHO, Geneva. 